\title{
II. Ein Fall von hinter dem Colon ascendens gelegenem Abscess.
}

Von Dr. C. Eisenlohr in Hamburg.

Pat, ein 37jähriger Ewerführer, wurde als notorischer Alkoholist zweimal auf der Alkoholistenabtheilung des allgemeinen Krankenhauses behandelt, zuerst vom 10. September bis 2. December vorigen Jahres, da er sich wegen hartnäckiger profuser Durchfälle, Neigung zu Blutungen aus der Nase, aufnehmen liess. Die Diagnose lautete: Cirrhosis hepatis mit Vergrösserung der Leber, Milzschwellung; Ascites war damals nicht nachweisbar, wohl aber leichter Icterus.

Im allgemeinen fieberfrei, machte Pat. während dieses ersten Aufenthaltes eine mehrtägige Fieberattacke mit Benommenheit des Sensorium und starken Durchfällen ohne positiven Localbefund durch, erholte sich aber rasch und ging Anfang December in verhältnissmässig gutem Zustande ab.

Am 14. Mărz dieses Jahres liess sich Pat. wieder aufnehmen; er war Tags zuvor unter Frost an Erbrechen und Durchfall mit starker Auftreibung des Leibes erkrankt; er machte bei ziemlich freiem Sensorium einen schweren Krankheitseindruck. Der Leib war stark meteoristisch aufgetrieben, doch weich und nur in der rechten hypochondrischen Gegend druckempfindlich. Freie Flüssigkeit im Abdomen nicht nachweisbar, die Grenzen der Leber und Milz wegen des Meteorismus nicht zu bestimmen. Leicht icterische Hautfärbung, mässig frequente grünschleimige Durchfälle. Erbrechen gallig, nicht fäculent. Mässiges remittirendes Fieber. Opiumbehandlung brachte Erbrechen und Durchfall bald zum Aufhören. Dagegen bestand Meteorismus mit starker Oppression, zeitweiser intensiver Schmerzhaftigkeit in der Regio hypochondr. rechts fort, ohne dass eine besondere Resistenzvermehrung oder gar ein Tumor fühlbar gewesen wäre.

Die Stühle waren gallig gefärbt; der Urin enthielt kein Eiweiss, aber (am 21. notirt) Pepton.

Am 24. März war zum ersten Mal Fluctuation im Abdomen nachweisbar; dagegen schwanden die peritonitischen Erscheinungen. Trot sehr günstiger Wirkung einer Copaivaemulsion auf die Diurese entwickelte sich im Laufe der nächsten Wochen ein beträchtlicher Ascites, dessen freie Beweglichkeit nur im Beginn durch Adhäsionen in der linken Reg. hypogastrica etwas beeinträchtigt schien.

Was dem Krankheitsbilde einen von dem gewöhnlichen Verlauf einer Lebercirrhose abweichenden Charakter verlieh, war das vom 28. März sich stärker erhebende, regelmässig morgens remittirende, abends gegen oder über 39 steigende Fieber, das bis zum 20. April andauerte, ohne speciellen localen Befund. Der Puls stets um 100. Von dem genannten Tage all blieb der Pat. afebril.

Der weitere Verlauf bot nichts ausserhalb des Krankheitsbildes deı letzten Stadien einer Lebercirrhose liegendes. Zweimalige Punction des Ascites verschaffte jeweils nur kurze Erleichterung, doch die Möglichkeit, die Grössenabnahme der ausserordentlich harten Leber zu constatiren.

In den letzten Wochen lag Pat. meist in einem halbstuporösen $\mathrm{Zu}$ stande, mit häufigen Klagen über Brustbeklemmung und quälenden Husten. Der Meteorismus blieb auch nach den Entleerungen des Ascites ziemlich beträchtlich; einmal erfolgte blutiges Erbrechen; der Urin enthielt in der letzten Zeit stets etwas Eiweiss. Am 29. Mai erfolgte der Tod.

Die Section (am 30. Mai) ergab eine mässig verkleinerte, sehr derbe, grob granulirte cirrhotische Leber, einen derben Milztumor und eine bindegewebige Induration des Pankreas.

Die V en a portae und Verzweigungen frei, ebenso die Gallenblase und das System der gallenführenden Gänge. Hinter dem oberen Abschnitt des Colon ascendens, zwischen Ligam. hepatico-colicum, vorderer Fläche der Nierenkapsel, absteigendem Theil des Duodenum, fand sich ein unit spärlicher eingedickter, breiiger, gelbgefärbter Masse gefüllter Herd, der ca. $10 \mathrm{~cm}$ im verticalen, etwas weniger im queren Durchmesser mass. Die Lage desselben entsprach dem Mesenterium der oberen Partie des Colon ascendens, die Wände des Herdes, also der untere Abschnitt des Lig. hepatico-colicum der Peritonealüberzug des Duodenum und die Nierenkapsel derb und stark bindegewebig verdickt. Das Peritoneum und der Peritonealüberzug des Darmes, spec. des Coecum wie des Processus vermifornis vollkommen frei, ohne Spur von Residuen einer Entzündung. Die Schleimhaut des Duodenum ohne Veränderung, im Jejunum und Ileum durchgängig frische zierlichı Injection der Schleimhautgefässe, im Colon transversum, descendens und der Flexur die Peyer'schen Plaques schwarzgrau gefärbt, aber nirgends ulcerirt oder narbig verändert. Ebenso zeigte sich die Schleimhaut des Coecum und Colon ascendens bei genauester Betrachtung ohne eine Spur von frischer oder älterer Continuitätstrennung, Narbenbildung oder Verdickung. Auch die übrigen Häute des betreffenden Darmabschnittes zeigten durchaus keine Veränderung der Structur; der beschriebene Herd lag vollständig ausserhalb der Darmwand, theils im Mesocolon ascendens, theils in retroperitonealen und prärenalen Gewebe.

Der Inhalt des Herdes bestand aus lebhaft gelbgefärbten, weichen Massen, die sich mikroskopisch als Fett, theils in grösseren rundlichen Kugeln, theils in körnigem Detritus erwiesen.

Es handelte sich zweifellos um einen älteren, grossentheils resorbirte oder fettig metamorphosirten Abscess, dessen Lage durch die gegebene Schilderung vollständig charakterisirt ist.

Was die Beurtheilung des Falles intra vitam anlangt, so war die Situation des Eiterherdes wegen des bestehenden hochgradigen Meteorismus und des circumscripten Umfangs, später wegen des die Lebercirrhose begleitenden Ascites nicht zu erkennen. Die im Krankheitsbild der Lebercirrhose intercurrirenden acuten Erscheinungen liessen wohl einen Abcess mit grosser Wahrscheinlichkeit annehmen (der Befund von Pepton im Urin war eine Stütze für diese Annahme), indess über die Natur und den Ausgangspunkt 
desselben war nicht völlig in's Klare zu kommen. Gegen die Annahme einer Pylephlebitis mit secundären Eiterherden der Leber sprach doch der Fiebercharakter, der Mangel von Frösten und der schliessliche Rūckgang der Erscheinungen. Eine zeitlang wurde an eine chronische hämorrhagische Peritonitis gedacht, doch besonders nach der Punction des Ascites, die ganz klare Flüssigkeit lieferte, der Gedanke fallen gelassen. Wir mussten uns schliesslich mit der allgemeinen Supposition einer circumscripten Peritonitis begnügen; eine vom Coecum oder Processus vermiformis ausgehende Affection war geradezu auszuschliessen.

Der Fall und das Prāparat scheint mir einiges Interesse zu verdienen, einmal als ein sehr sauberes Beispiel eines Processes, wie er häufig in der Umgebung des Coecum als eigentliche Perityphlitis vorkommt, hier aber localisirt auf die Gegend des Colon ascendens.

Die anatomische Beurtheilung ist bei den Processen der Typhlitis und Perityphlitis meist durchaus keine einfache und leichte, weil die Veränderungen häufig relativ alte sind, und primāre und secundäre Entzündıngsvorgänge, intra- und extraperitoneale Herde sich compliciren.

Für die perityphlitischen Processe ist allgemein zugegeben, dass der häufigste und gewônhliche Ausgangspunkt der Processus vermiformis ist, und die ursächlichen Momente, die zur Erkrankung dieses Darmabschnittes führen (Obstruction, Nekrose etc.), sind hinlänglich bekannt. Aber schon die nächsten Folgen der partiellen Nekrose dieses Darmtheils sind noch keineswegs in allen Modalitäten klargelegt, am Sectionstisch recht schwer und in vivo meist gar nicht zu definiren. Und wenn auch speciell die letzten Jahre eine Reihe von Stndien über die Formen der Typhlitis speciell von amerikanischen nnd französischen Autoren gebracht haben, wenn man auch die Entrinndnng des Wurmfortsatzes, die Appendicitis schärfer zu trennen versucht hat von gewissen Fällen primärer perityphlitischer Erkranknng (Bucquoy und Dantel), wenn man auch die Folgen der Perforation des Wurmfortsatzes, je nachdem letztere in sein Mesenterium oder ausserhalb desselben stattfindet, schärfer getrennt hat (Porter), so ist ein Auseinanderhalten dieser Modificationen und Vorgänge in der klinischen Beobachtung kaum moglich.

Unser Präparat giebt einen unzweifelhaften Beweis dafür, dass circumscripte , colitis ch $\mathrm{e}^{\text {" }}$ Herde ohne Betheiligung der eigentlichen Peritonealhöhle, wiewohl mit peritonitischer Reizung und mit starker Verdickung peritonealer Falten und Ligamente vorkommen, dass diese Abscesse - um einen solchen im Stadium der Resorption handelte es sjch jedenfalls - aber auch vorkommen, ohne dass im Darm ulcerative Processe oder gar nekrotische Zerstorung der Darmwand vorausgegangen. Wie unser Präparat lehrt, ist das Mesenterium resp. das Mesocolon an umschriebener Stelle der Sitz solcher Abscesse; von da verbreiten sie sich in das retroperitoneale, prärenale Bindegewebe. Dass der Ausgangspunkt doch eine vielleicht ganz oberflächliche Continuitätstrennung der Schleimhaut in dem correspondirenden Darmabschnitt (Colon ascendens) gewesen sein muss, ist mehr wie wahrscheinlich.

Der Sitz des Herdes giebt mir Veranlassung, an einzelne Fälle meiner Beobachtung zu denken, die unter der Signatur Perityphlitis gingen, in ihrem Verlauf auch durchaus dieser Signatur entsprachen, iu denen aber der von vornherein palpable empfindlich-elastische Tumor nicht eigentlich dem Coecum, sondern einer höheren Partie des Colon ascendens entsprach. Wahrscheinlich sind diese Fãlle von Colitis oder Pericolitis ascendens nicht so selten, und das Präparat ist ein anatomischer Beweis für ihr Vorkommen. Dass hier Coecum und Wurmfortsatz nie im geringsten betheiligt waren, brauche ich nicht speciell zu wiederholen. Der Palpation war der Herd wohl deshalb nicht zugänglich, weil ein sehr beträchtlicher Grad von Meteorismus (später Ascites) bestand, und seine Lage ohnehin die Palpation erschwerte.

Die gefundenen Residuen zeigen, dass solche Eiterherde sich wirklich vollommen ohne Entleerung resorbiren kōnnen; der spārliche Inhalt des Herdes bestand nur aus fettigem Detritus und enthielt keine Eiterkörperchen mehr. Symptome hatte der Herd in der letzten Zeit anch nicht mehr gemacht. 